\title{
Pediatric Phenylketonuria in Fayoum Governorate (Retrospective study)
}

\author{
EssaamEldeen Gad Elrab Ahmed ${ }^{(1)}$, Ashraf Sayed Kamel ${ }^{(2)}$, Mohamed Masoud \\ Said $^{(3)}$,Fatma Said Abdeltwab Hassan ${ }^{(4)}$. \\ (1) Resident of pediatrics - fayoum Health insurance hospital. \\ (2) Professor of pediatrics-Faculity of medicine-Fayoum University. \\ (3) AssProfessor of Pediatrics-Faculity of medicine-Fayoum University. \\ (4) Lecturer of public health and community medicine Faculty of Medicine-Fayoum \\ university.
}

Corresponding author: Fatma Said Abdeltwab Hassan

E mail address: elzahraa.elashery@yahoo.com

Tel: 01024556995

\section{ABSTRACT}

Aim of work: The aim of this study is to explore epidemiological and clinical situation of phenylketonuria among children in Fayoum governorate and to assess the current state of PKU screening and management.

Patients and Methods: This study was conducted with 2 approaches; a retrospective approach related to the recorded data of phenylketonuria cases on Fayoum governorate and a cross sectional approach to complete history taking and examination of the cases.

Results: From 9/2015 to 9/2017, 229547 newborns in Fayoum were screened for PKU with screening coverage rate of $98.8 \%$. Out of 61 mothers of the 71 PKU cases, 49/61 $(80.3 \%)$ reported consanguineous marriage, $19 / 22(86.5 \%)$ of them showed

\section{INTRODUCTION}

Phenylketonuria (PKU) is an autosomal recessive metabolic disorder characterized by a dysfunctional or absent enzyme, phenylalanine hydroxylase (PAH), which is responsible for the metabolism of the amino acid phenylalanine (Phe). With little to no functional PAH, Phe accumulates in the blood and brain of individuals with PKU (De Groot et al ., 2010) $)^{(2)}$. appearance of PKU (fair complection of skin, hair and iris), As regards neurological examination, 13/65 (20.0\%) had delayed speech.

Conclusion and recommendations:

Incidence of PKU among screened neonate in Fayoum governorate was high which implicates the importance of NBS program for early detection of PKU cases before appearance of manifestations.

Consanguinous marriage was found in most cases of PKU. Health education specially for those living in rural area as regard the role of consanguineous marriage and family history as a risk factor of PKU is an important issue.

KEY WORDS: phenylketonuria, phenylalanine, PHE free formula, epidemiology of PKU.

Newborn screening (NBS) is a public health measure aimed at identification of early cases, management of afflicted infants, and making efforts to reduce morbidity and mortality among newborns. NBS is a comprehensive system of education, screening, follow-up, diagnosis, treatment/management, and evaluation, which should be institutionalized and 
consolidated in the public health system of a

\section{PATIENTS AND METHODS:}

This study was conducted with 2 approaches; a retrospective approach related to the recorded data of all phenylketonuria cases registered in Fayoum governorate (74 cases), in the period from September 2015 to September 2017, and a cross sectional approach to complete history taking (from 71 cases) and examination of the cases (65 cases).

\subsection{Study population:}

The study included cases diagnosed as phenylketonuria according to the following eligibility criteria.

\section{Inclusion criteria:}

- $\quad$ All Cases of PKU diagnosed in Fayoum governorate either diagnosed by national newborn screening program or diagnosed lately after suspicion clinically or by family history.

- In the period from September 2015 to September 2017.

\section{Exclusion criteria:}

- Cases diagnosed in Fayoum and not belong to Fayoum governorate.

- Cases diagnosed before or after the period between September 2015 to September 2017.

- Cases aged more than eighteen years old.

\subsection{Data collection:}

country (Padilla et al., 2010) $)^{(5) .}$

Data were collected by 3 methods as shown in figure (1)

1. Available records of all cases $(\mathbf{N}=\mathbf{7 4})$ as regards:

- Method of discovering (from screening program or late diagnosis)

- Socio-demographic data

- Age at diagnosis

- Serial laboratory investigations of phenylalanine and tyrosine levels

2. Pre-designed structured Questionnaire : form for mothers of cases $(\mathbf{N}=\mathbf{7 1})$, either by interview with mother or by telephone, which covered;

- Data to confirm data collected from records; Socio-demographic, age at diagnosis

- Perinatal history

- Complaint at diagnosis \& developmental history

- Neurological history

- Nutritional history

- Data about feeding and growth situation .

3. The cases who could reach $(\mathbf{N}=\mathbf{6 5})$ were subjected to examination by the investigator and observed manifestations were written down in an examination template including;

- General examination \& anthropometric measurements.

- Systemic examination. 


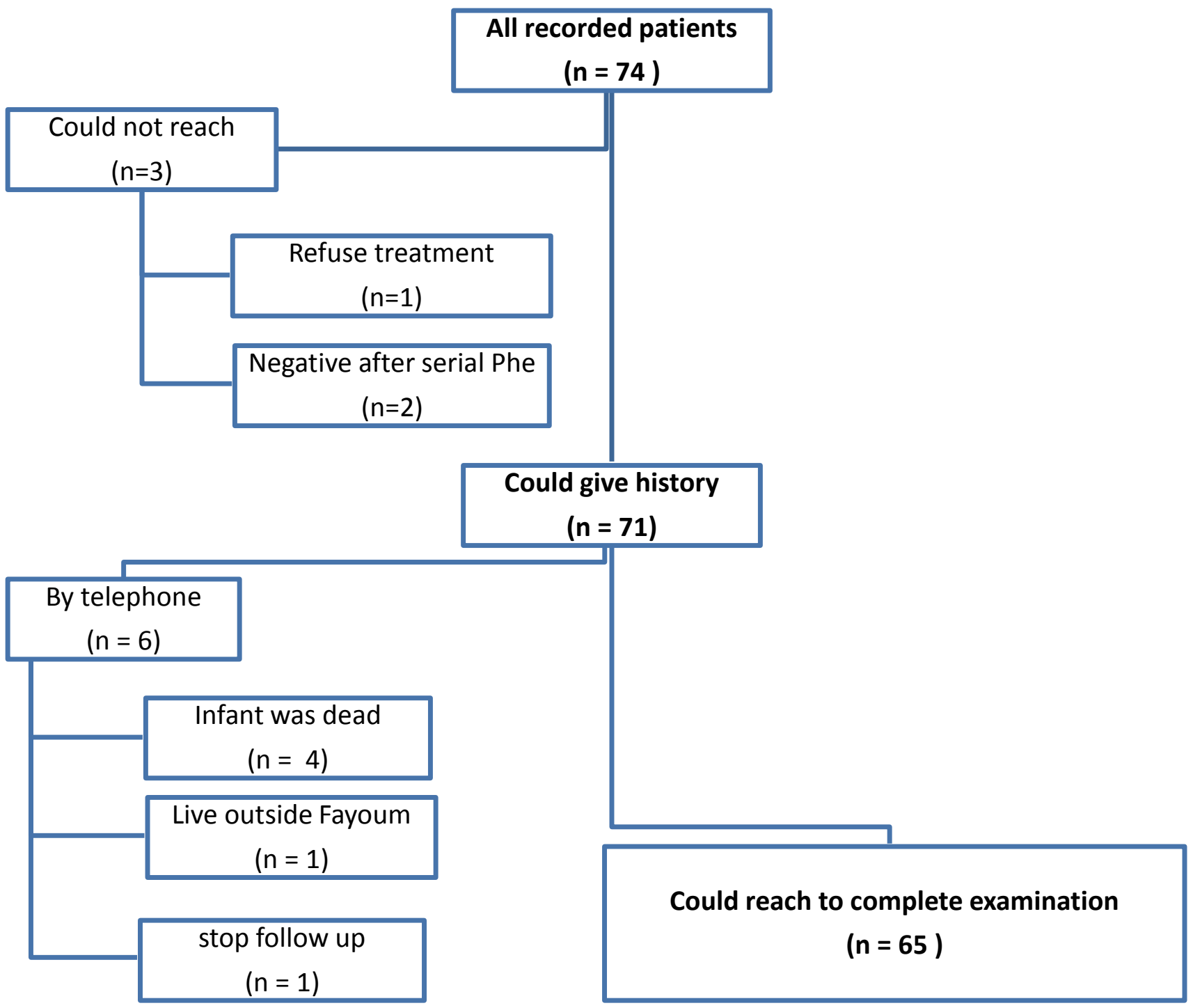

Figure (1) Flow chart showing study patients and different data collection methods and $\underline{\text { tools }}$

\section{RESULTS}

This study included children diagnosed as PKU and recorded in the national registry of PKU in Fayoum governorate from (9/2015 to 9/2017). The results of this work will be presented into three sections:

Section I: Recorded data of all registered cases ( 74 cases of PKU).
Section II: History taking from 71 cases of PKU.

Section III: Clinical examination of 65 cases of PKU.

3.1 incidence:From 9/2015 to 9/2017, 229547 newborns in Fayoum were screened 
for PKU with screening coverage rate of $98.8 \%$. Out of them, 59 were diagnosed as PKU with incidence rate of 2.6 per 10000. Incidence rate during 2015, $2016 \& 2017$ was 2.1, $2.6 \& 2.8$, respectively. Median age of diagnosis for screened neonates was 7

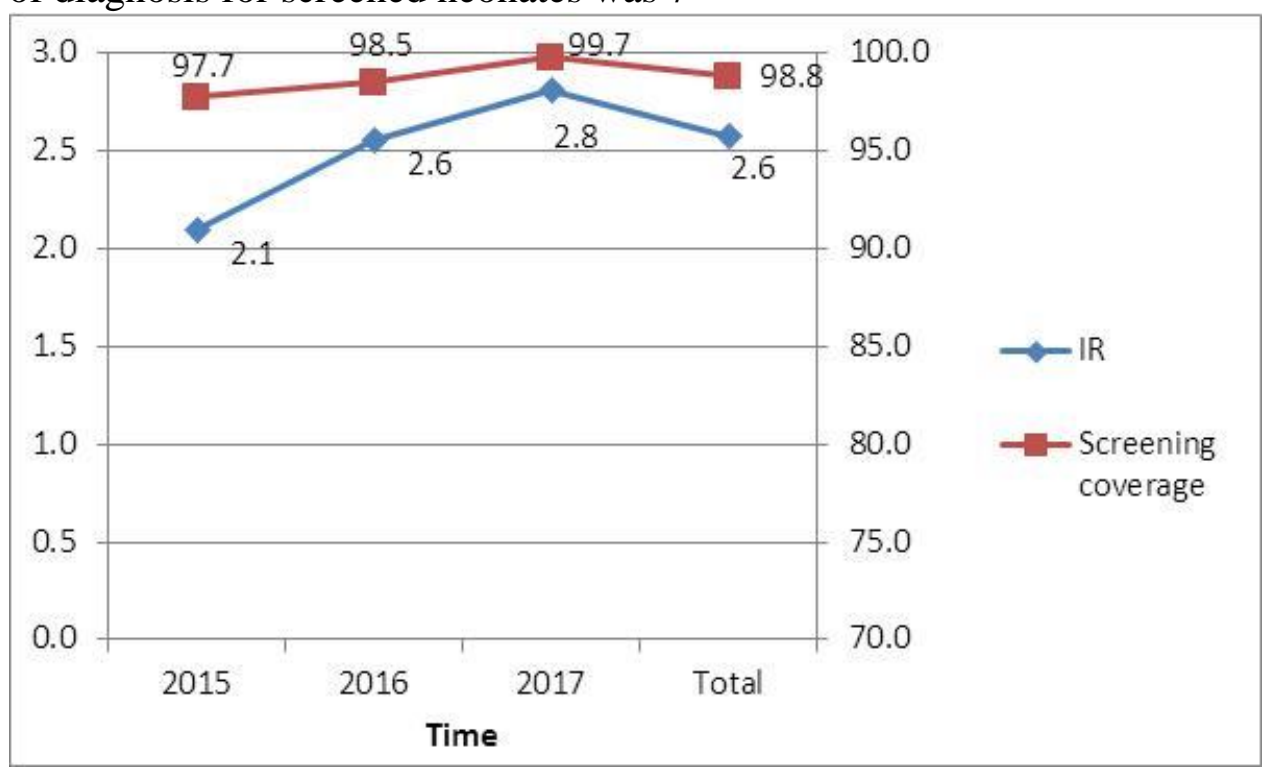

Figure (2) Coverage of PKU neonatal screening and incidence of PKU among screened neonates

\subsection{Socio-demographic characteristics of children with PKU (N=74)}

Males 39/74 (52.7\%) were more than females $35 / 74(47.3 \%)$ with male to female ratio $=1.1: 1$. Median age at time of the study is 1.5 years with mean $\pm \mathrm{SD}$ of $2.4 \pm 2.9$. As regard of residence, 71/74 (95.9\%) were from rural area while $3 / 74(4.1 \%)$ were from urban area. Most cases lived in Etsa District $33(44.6 \%)$ following by Tammyia 15 (20.3\%), Sinoris $12(16.2 \%)$ and 6 cases $(8.1 \%)$ reported from Fayoum and Yousef El sedique. Only, 2 cases (2.7\%) reported from Abshawi. days with mean \pm SD of $8.1 \pm 3.3$.

Moreover, during this period, 15 cases were diagnosed as PKU very late. Their median age was 6 years with mean \pm SD of $7.1 \pm 5.1$, figure (2). 


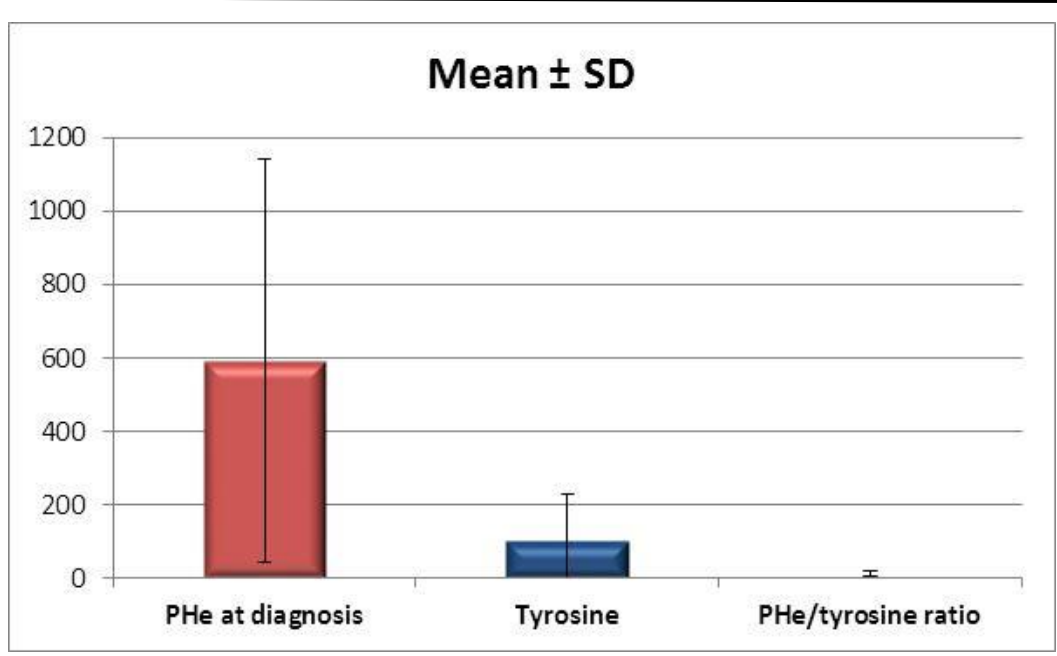

Figure (3) PHe, tyrosine and PHe/tyrosine ratio at diagnosis of children with PKU

3.5 As regarding motor developmental history, 28/71 (39.4\%) patients had history of motor delay. Delayed head support was reported in $21 / 71$ (29.6\%), delayed sitting in $28 / 71(39.4 \%)$, delayed crawling in 25/71 (35.2\%), delayed standing 23/71 (32.4\%), delayed walking in $17 / 71(23.9 \%)$.

3.6 According to mental developmental history, 23/71 (32.4\%) patients had history of mental delay. Delayed social smile was reported in $11 / 71(15.5 \%)$, delayed recognition of mother in 18/71 $(25.4 \%)$.

3.7 History of association with other diseases was positive in $1 / 71(1.4 \%)$ because of hypothyroidism

3.8 The data revealed that $15 / 71(25.1 \%)$ of patients had history of convulsion with $9 / 15$ (60\%) had history of repeated convulsions while 6/15 (40\%) experienced one attack of convulsion. Also, Abnormal movements was found in 7/71 (9.9\%) of patients. As well as, behavioral disorders was reported in 39/71 $(54.9 \%)$. The most frequent behavioral disorders were nervousness 23/37 (59.0\%), nervousness \& excessive crying 9/37 (23.0 $\%$ ), autism in $2.6 \%$ and ADHD in $2.6 \%$.

3.9 As regarding history of manifestations of skin and soft tissue; eczema was found in
$36 / 71(50.6 \%)$ of patients. Also, fair skin was reported in 27/71 (38.0\%) of cases. Iris color was blue, green and grey in 21/71 (29.6\%) of cases. As well, 50/71 (70.4\%) of cases had history of musty odor

3.10 nutritional disorders and feeding related problems were found in 13/71 (19.3\%) of cases; in the form of repeated vomiting in $8 / 13(61.5 \%)$, anemia in $4 / 13(30.8 \%)$, and hypocalcemia in 1/13 (7.7\%). Regarding weight gain, $6 / 71(8.5 \%)$ of cases failed to gain their weight, Teething normal in $74.6 \%$ and in $25.4 \%$ not yet (i.e not teething delay).

3.11 Breast feeding was reported in 54/71 (76.1 50), artificial feeding (standard formula) was stated in 16/71 (22.5\%), mixed breast and artificial was found in 1/71 (1.4\%), 70/71 (98.6\%) had history of $\mathbf{3 . 1 2}$ PHE free formula intake; 5/70 (7.1 5) of cases discontinued due to improvement, while $2 / 70(2.9 \%)$ discontinued due to incompliance. As regarding to low PHE diet, it was reported in 47/71 (66.2\%) of cases. As well, 19/71 (40.8\%) had history of taking nutritional supplementation in the form of iron, vitamin D and calcium as in figure 4 


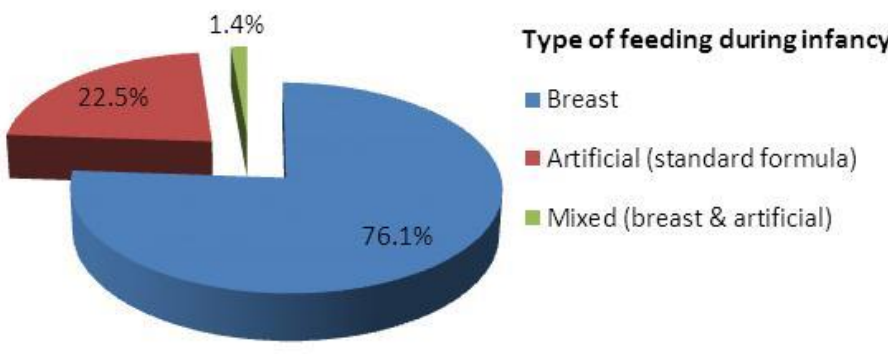

Figure (4): Type of feeding during infancy in children with PKU ( $N=71$ ).

\section{DISCUSSION}

4.1 Incidence rate in fayoum governorate during 2015, $2016 \& 2017$ was 2.1:10000, 2.6:10000\& 2.8:10000, respectively while identified incidence rate in the country as a whole by 1 per 7500 (i.e. 1.3 in 10000) from the start of national newborn screening program so fayoum governorate has a high incidence rate while Scriver and Kaufman., $2001^{(6)}$ reported incidence of PKU by population, the highest incidence in turkey (2.6 : 10000), then Yemenite Jews (in Israel $1,8: 10000$ ) then Arabic population (up to $1: 6000)$ and the lowest incidence in Finland $(0,05: 10000)$, Japan $(0,08: 10000)$, in comparison to our study Fayoum are in average incidence as in turkey.

\subsection{Cases who diagnosed lately in this} current study were 15 , the diagnosis occurred after diagnosis of relatives (sister, brother, cusins ) by NBS in most of cases (10 cases ) which describe the importance of NBS, Past history of neurological drugs administration specialy who lately diagnosed as they almost misdiagnosed as cerebral palsy or epilepsy. The same situation occur in the study conducted by Eshraghi et al., $\mathbf{2 0 1 0}^{(3)}$ who reported that The main reason behind delayed diagnosis in the Mexican study was reported to be the absence of routine screening programs in the health care system. After 2006, a national routine screening program was included for PKU in Mexico after which the number of earlydetected patients noticeably increased.

4.3 In our study there was association of PKU with behavioral abnormalities as autism, while Gillberg et al., $2016^{(4)}$ reported that there is a need for caution in interpreting the significance of similarities or co-morbidities among autism spectrum disorder (ASD) and the behavioral phenotypes of ASD and PKU.

4.4 As regard to type of feeding during infancy : breast feeding was $76.1 \%$, artificial feeding (standard formula) was $22.5 \%$, mixed breast and artificial was $1.4 \%$. This is not in agreement with Banta-Wright et al., $2012^{(1)}$ who reported that diet has been the main focus of treatment and mothers were advised against breastfeeding infants, although breast milk is lower in phenylalanine than most formulas. 


\section{CONCLUSIONS}

This study revealed the following conclusions:

Incidence of PKU among screened neonate in fayoum governorate was high in comparison of the country as a whole, The coverage of the neonatal screening for PKU was high and showed no change in its strength by time, Consanguinous marriage was found in most cases of PKU and all families included in family pedigrees revealed consanguinity, The most frequent complaints among those with delayed diagnosis were mental and motor delay, The

\section{ACKNOWLEDGEMENTS}

This work was supported by Fayoum health directorate for agreement to see records and supported by urban health center in fayoum

\section{ABBREVATIONS}

ASD: autism spectrum disorder, NBS: Newborn screening, PHE :

Phenylalanine,

\section{REFRENCES}

\section{[1] Banta-Wright SA, Shelton KC, Lowe}

ND et al (2012). Breast-feeding success

among infants with phenylketonuria. $J$

Pediatr Nurs. ; 27(4):319-327.

[2] De Groot M.J., Hoeksma M., Blau N., Reijngoud D.J., Van Spronsen F.J., (2010) :Pathogenesis of cognitive dysfunction in phenylketonuria: review of hypotheses, Mol. Genet.Metab. 99 S86-S89.

\section{[3] Eshraghi P, Abaskhanian A, Mohammadhasani A (2010):}

Characteristics of patients with phenylketonouria in Mazandaran Province, most frequent symptoms of motor delay were delayed sitting, crawling and standing, Abnormal behavioural disorders such as nervousness and crying was reported in most cases of PKU, Musty odour and eczema were the most common skin and sof tissue manifestations, Near half of PKU cases had length/height less than $5^{\text {th }}$ percentile, Majority of PKU cases reported intake of PHE free formula with good compliance. In contrast, small proportion of PKU cases refused intake of PHE free formula or discontinued due to poor compliance.

No funding as its retrospective study depended on history taking and physical examination and avaliability of data in records.

PAH : Phenylalanine hydroxylase enzyme, PKU : phenylketonuria, SD: Standard deviation

Northern, Iran. Caspian Journal of Internal Medicine. ; 1(2): 72-4.

[4] Gillberg C, Coleman M. Mitchell JJ, Trakadis YJ, Scriver CR(2016): Autism Spectrum Disorder and Phenylketonuria: Dyzygotic Twins with Double Syndrome Arch Neuropsychiatry 2017; 54: 92-3 • DOI: 10.5152/npa.2016.12500.

\section{[5] Padilla CD, Krotoski D, Therrell BL}

$\mathbf{J r}(\mathbf{2 0 0 9 )}$ : Newborn screening progress in developing countries - overcoming internal barriers. Semin Perinatol. 2010; 34(2): 145- 
55. doi: 10.1053/j.semperi..12.007. PMID: 20207264.

[6] Scriver CR, Kaufman S.

Hyperphenylalaninemia (2001):

phenylalanine hydroxylase deficiency. In:
Scriver CR, Beaudet AL, Sly WS, Valle D, Childs B, Kinzler K, Vogelstein B, editors. The Metabolic and Molecular Bases of Inherited Disease. 8th ed. New York: McGraw- Hill;. p. 1667-724. 